\title{
Bilateral Phyllodes Tumor of the Breast; a Case Report of Benign Tumor on One Side and Malignant Tumor on the Contralateral Side
}

\author{
Hamdy Abdel Azim 1, Omar Abdel-Rahman ${ }^{2}$, Raafat Abdel-Malek ${ }^{1}$ \\ ${ }^{1}$ Clinical Oncology department, Kasr Al-Ainy School of medicine, Cairo University, Cairo, Egypt; \\ ${ }^{2}$ Clinical Oncology department, Faculty of Medicine, Ain-Shams University, Cairo, Egypt
}

Introduction: Phyllodes tumors (PTs) of the breast are rare fibroepithelial neoplasms which may be benign, borderline or malignant. Malignant PTs tends to recur more frequently and has the potential to metastasize. Bilateral PT is a rare presentation of the disease.

Case presentation: We report a case of 34 years old female patient with bilateral breast PT, malignant on one side and benign on the contralateral one. First, she underwent lumpectomy for right breast benign PT which recurred six month later and underwent right mastectomy. One year later, she underwent excision of left breast borderline PT which recurred six months later. The recurrence was in the form of malignant PT for which she underwent mastectomy. Almost one year later, she developed right chest wall recurrence and was found to have bone, brain and lung metastases. She received one cycle of ifosfamide plus doxorubicin with no noticeable response after which she deteriorated rapidly and died few weeks later. Discussion: Bilateral breast PT is very rare and its management represents a challenge in view of limited evidence.

Keywords: Phyllodes tumor, Breast, Bilateral, Metastases

Corresponding author: Raafat Abdel-Malek, MD; Clinical Oncology Department, Kasr Al-Ainy School of Medicine, Cairo University, Cairo, Egypt; Email: raafat.malek@kasralainy.edu.eg

Received: 1-December-2016, Revised: 5-Septebmer-2017, Accepted: 5-September-2017, Published online: 10-September-2017

\section{INTRODUCTION}

Phyllodes tumors (PTs) of the breast are rare fibroepithelial neoplasms, representing $0.3-1 \%$ of all primary breast tumors and $2.5 \%$ of breast fibroepithelial tumors. Phyllodes tumors are usually benign and occur in middle-aged women with an average age ranging from 40 to 50 years ${ }^{1}$. The German Physician Johannes Müller was the first to describe PTs in 1838 and coined the term "cystosarcoma phyllodes" which was found later to be an inaccurate description of the tumor because it is usually benign ${ }^{2}$.

Histologically, it is described as biphasic fibroepithelial lesion of the breast, characterized by leafy stromal fronds capped by benign bilayered epithelium ${ }^{3}$. The World Health Organization classified PTs into three subgroups; benign, borderline, and malignant. This classification is based on histologic features that include stromal hypercellularity, cellular pleomorphism, mitosis (per $10 \mathrm{HPF}$ ), tumor margin and stromal pattern ${ }^{1}$. Phyllodes tumors are benign in $60 \%-75 \%$ of cases, borderline in $12 \%-26 \%$ and malignant in $10 \%-15 \%{ }^{4}$.

A number of reports described bilateral breast PTs which is an uncommon presentation of this relatively rare tumor ${ }^{5-10}$. We report here the case of a 34 years old female patient diagnosed with bilateral PT of the breast; benign tumor on one side and malignant tumor on the contralateral side metastatic to the brain, bone and lung.

\section{CASE PRESENTATION}

A 34 years old female patient first presented in February 2006 with right breast lump. Bilateral mammography revealed right breast lump in the upperouter quadrant. She underwent lumpectomy in March 2006 and the histopathological examination revealed benign PT. Six months later in September 2006, she developed local recurrence for which she underwent simple mastectomy. The histopathological evaluation revealed borderline PT which was strongly positive for estrogen receptors (ER) and progesterone receptors (PR). The patient was then kept under follow up.

In October 2007, the patient complained of left breast mass. Excision biopsy and histopathological examination revealed borderline PT. Six months later, in April 2008, the patient developed local recurrence in the left breast for which she underwent left modified radical mastectomy. Histopathological examination revealed malignant PT. She was then kept under regular 3monthly follow up.

In May 2009, she developed right chest wall local recurrence. Positron emission tomography-computed tomography (PET-CT) scan revealed right breast local recurrence associated with bone, brain and lung metastases (figure 1). Biopsy from a paravertebral mass revealed metastatic spindle cell carcinoma, grade 2 . Immunohistochemical findings were compatible with metastatic sarcomatous element of malignant PT. As the patient's Eastern Cooperative Oncology Group (ECOG) performance score was 2 , she was offered chemotherapy (ifosfamide + doxorubicin). Unfortunately, progression in the size of the chest wall mass was noted after the first cycle together with deterioration in her performance status. Systemic chemotherapy was discontinued and she received best supportive care only until her death few weeks later. 

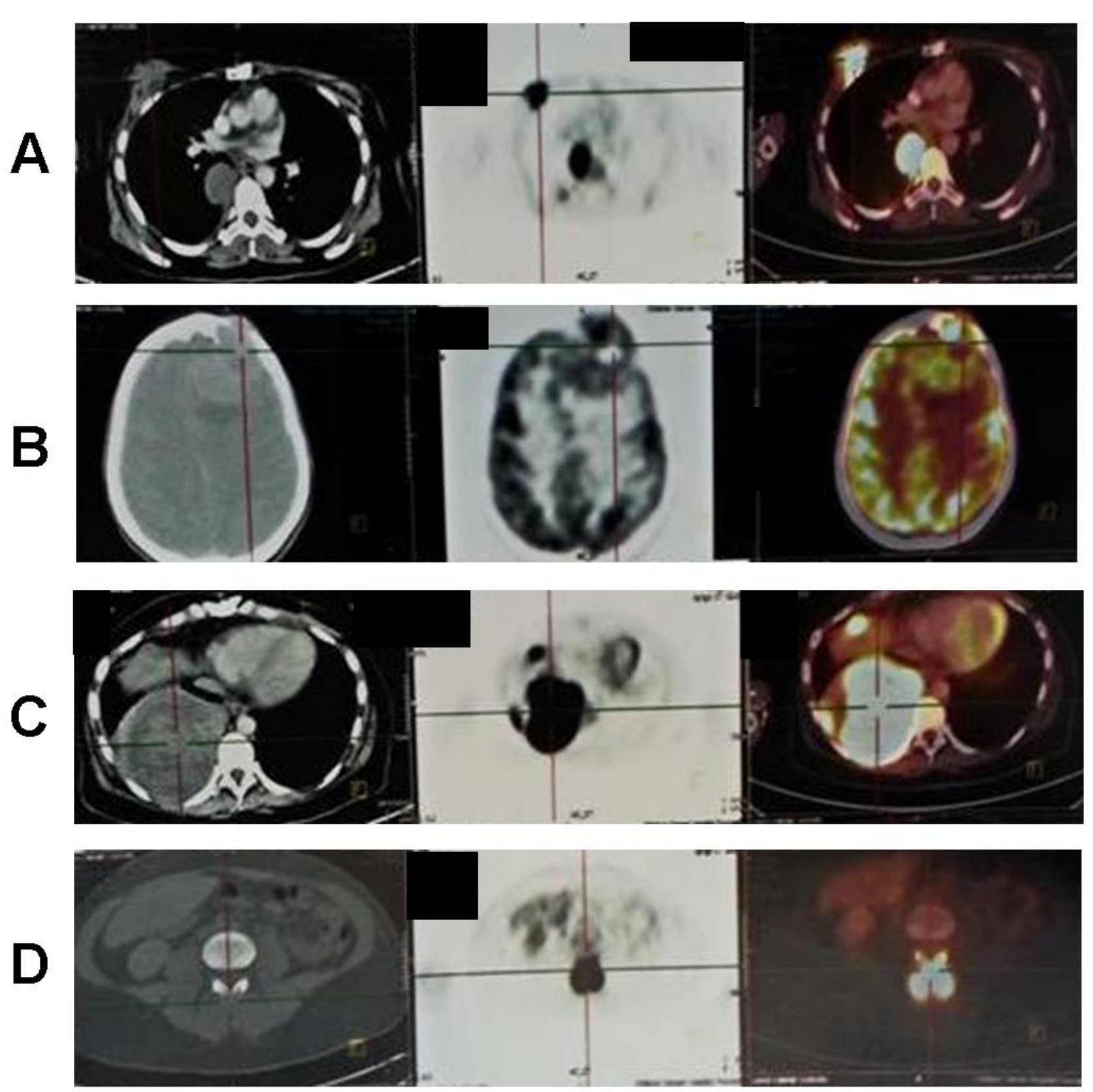

Figure 1. Positron emission tomography-computed tomography (PET-CT) scan images showing: A- Right chest wall recurrence, B- Brain metastasis, C- Lung metastasis and D- Bone metastasis

\section{DISCUSSION}

Overall, the prognosis of PTs is favorable and depends on the histopathological subtype. The local recurrence rate is around $20 \%$ in benign PT and is up to $25 \%$ in borderline PT. In malignant PT, the recurrence rate may as high as $40 \%$ and distant metastases may occur in $9 \%$ to $27 \%$ of cases ${ }^{4}$.

The bilateral synchronous or metachronous representation of breast PTs is very rare. Case reports describing bilateral PTs are usually of the benign subtype ${ }^{5-7}$. Less commonly, bilateral malignant PTs were reported ${ }^{8,9}$. Only one case of bilateral PT with malignant subtype on one side and benign subtype on the contralateral side was reported by Mrad et al in $200{ }^{10}$.
To the best of our knowledge, the current case is the second to be reported with malignant PT and contralateral benign PT.

In the current case, before being metastatic, the only treatment modality was surgery. Although surgery has been long advocated as the main line of treatment for localized PTs of the breast, the type of surgery and the adequate margin width has been debated ${ }^{11}$. The current evidence suggests that there is no difference in the recurrence rate between $1 \mathrm{~mm}$ and $10 \mathrm{~mm}$ surgical margin for benign PTs ${ }^{12}$. In malignant PTs, excision with at least $10 \mathrm{~mm}$ negative margin or mastectomy is recommended ${ }^{2}$.

Because of the lack of strong evidence to use adjuvant therapy routinely in PTs, our patient did not 
receive radiotherapy, chemotherapy or hormonal therapy following surgery. Although there are no randomized trials to support its benefit, post-operative radiotherapy for all malignant PTs has been advocated by some investigators. Some even suggested post-operative radiotherapy for selected cases of borderline PTs ${ }^{2,11}$. On the other hand, multiple chemotherapy regimens has been tested in the adjuvant setting of malignant PT with no progression-free or overall survival benefit $2,13,14$. Despite the high ER content of the epithelial component of most PTs, adjuvant endocrine therapy failed to demonstrate a role in the treatment of PTs of the breast 11,15 .

Malignant phyllodes tumors may give rise to haematogenous metastases in up to $20 \%$ of cases, most frequently to the lungs, abdominal viscera and bones ${ }^{2}$, 11. The prognosis of metastatic malignant PT is dismal. In the rare setting of metastatic malignant PTs, like in our case, it is recommended to follow guidelines for the management of metastatic soft tissue sarcoma ${ }^{16}$. The systemic treatment that we used in the current case (ifosfamide and doxorubicin combination chemotherapy) is among the recommended regimens ${ }^{17}$. However, the aggressive tumor of this case did not show response and the patient deteriorated rapidly which precluded trying different systemic therapy protocols.

In conclusion, bilateral representation of PTs is rare and the current case is among the few reported. In view of the limited evidence available, the management of such cases is quite challenging.

\section{REFERENCES}

1. Bellocq JP, Magro G. Fibroepithelial tumours. In: Tavassoli FA, Devilee P (eds). World Health Organization classification of tumours: Pathology and genetics of tumours of the breast and female genital organs. Lyon, France: International Agency for Research on Cancer Press, 2003.

2. Strode M, Khoury T, Mangieri C, Takabe K. Update on the diagnosis and management of malignant phyllodes tumors of the breast. Breast. 2017; 33: 91-96.

3. Tan PH, Thike AA, Tan WJ, et al. Predicting clinical behavior of breast phyllodes tumours: a nomogram based on histological criteria and surgical margins. J Clin Pathol. 2012; 65(1): 69-76.
4. Zhang Y, Kleer CG. Phyllodes Tumor of the Breast: Histopathologic Features, Differential Diagnosis, and Molecular/Genetic Updates. Arch Pathol Lab Med. 2016; 140(7): 665-671.

5. Singh J, Singh B, Chander J. Extramammary cystosarcoma phyllodes with bilateral breast involvement. Aust N Z J Surg. 1980; 50(1): 56-58.

6. Ezeome ER, Okafor OC, Nwajiobi CE, Osuagwu CC. Bilateral benign phyllodes tumour in a nulliparous woman: a case report and review of literature. Niger $\mathbf{J}$ Clin Pract. 2007; 10(1): 66-69.

7. Seal SK, Kuusk U, Lennox PA. Bilateral and multifocal phyllodes tumours of the breast: A case report. Can J Plast Surg. 2010; 18(4): 145-146.

8. Bader E, Isaacson C. Bilateral malignant cystosarcoma phyllodes. Br J Surg. 1961; 48: 519-521.

9. Ball $\mathrm{AB}$, Fisher C. Bilateral malignant phyllodes tumours. J Surg Oncol. 1991; 46(1): 67-69.

10. Mrad K, Driss M, Maalej M, Romdhane KB. Bilateral cystosarcoma phyllodes of the breast: a case report of malignant form with contralateral benign form. Ann Diagn Pathol. 2000; 4(6): 370-372.

11. Tan BY, Acs G, Apple SK, et al. Phyllodes tumours of the breast: a consensus review. Histopathology. 2016; 68(1): $5-21$.

12. Shaaban M, Barthelmes L. Benign phyllodes tumours of the breast: (Over) treatment of margins - A literature review. Eur J Surg Oncol. 2017; 43(7): 1186-1190.

13. Morales-Vásquez F, Gonzalez-Angulo AM, Broglio K, et al. Adjuvant chemotherapy with doxorubicin and dacarbazine has no effect in recurrence-free survival of malignant phyllodes tumors of the breast. Breast J. 2007; 13(6): 551-556

14. Haberer S, Laé M, Seegers V, et al. Management of malignant phyllodes tumors of the breast: the experience of the Institut Curie. Cancer Radiother. 2009; 13(4): 305312.

15. Tse GM, Lee CS, Kung FY, et al. Hormonal receptors expression in epithelial cells of mammary phyllodes tumors correlates with pathologic grade of the tumor: a multicenter study of 143 cases. Am J Clin Pathol. 2002; 118(4): 522-526.

16. National Comprehensive Cancer Network. NCCN Clinical practice guidelines in oncology: breast cancer, version 2.2017. Available from: https://www.nccn.org/professionals/physician_gls/f_guide lines.asp

17. National Comprehensive Cancer Network. NCCN Clinical practice guidelines in oncology: soft tissue sarcoma, version 2.2017. Available from: https://www.nccn.org/professionals/physician_gls/f_guide lines.asp 